\title{
Neutron scattering studies on protein dynamics using the human myelin peripheral membrane protein P2
}

\author{
Saara Laulumaa ${ }^{1,2, a}$, Petri Kursula ${ }^{1,2,3}$ and Francesca Natali ${ }^{4,5}$ \\ ${ }^{1}$ Faculty of Biochemistry and Molecular Medicine \& Biocenter Oulu, University of Oulu, Oulu, Finland \\ ${ }^{2}$ Centre for Structural Systems Biology, Helmholtz Centre for Infection Research (CSSB-HZI), German Electron \\ Synchrotron (DESY), Hamburg, Germany \\ ${ }^{3}$ Department of Chemistry, University of Hamburg, Hamburg, Germany \\ ${ }^{4}$ Institut Laue-Langevin (ILL), 71 avenue des Martyrs, CS 20156, 38042 Grenoble Cedex 9, France \\ ${ }^{5}$ CNR-IOM, OGG, 71 avenue des Martyrs, CS 20156, 38042 Grenoble Cedex 9, France
}

\begin{abstract}
Myelin is a multilayered proteolipid membrane structure surrounding selected axons in the vertebrate nervous system, which allows the rapid saltatory conduction of nerve impulses. Deficits in myelin formation and maintenance may lead to chronic neurological disease. P2 is an abundant myelin protein from peripheral nerves, binding between two apposing lipid bilayers. We studied the dynamics of the human myelin protein P2 and its mutated P38G variant in hydrated powders using elastic incoherent neutron scattering. The local harmonic vibrations at low temperatures were very similar for both samples, but the mutant protein had increased flexibility and softness close to physiological temperatures. The results indicate that a drastic mutation of proline to glycine at a functional site can affect protein dynamics, and in the case of P2, they may explain functional differences between the two proteins.
\end{abstract}

\section{Introduction}

The myelin sheath is an insulating, tightly packed membrane multilayer structure surrounding selected nerve axons. It protects and enhances transmission of nervous signals in the central (CNS) and peripheral nervous systems (PNS).

Myelin has a unique structure and contains mostly lipids $(75-80 \%)$, very little solvent and myelin-specific proteins at high concentrations. The role and function of myelin proteins remain poorly understood in both healthy myelin and as targets for several neurological diseases $[1,2]$. To understand myelin breakdown in pathology, it is of utmost importance to achieve a better knowledge of the parameters determining assembly and compaction of the myelin sheath [3].

Myelin protein P2 is one of the major components of human peripheral nerve myelin. It is a small $15-\mathrm{kDa}$ fatty acid binding protein (FABP) present mainly in compact myelin constituting up to $15 \%$ of the total protein fraction locally [4-6]. The crystal structure of human P2 has been solved at high resolution (Fig. 1) [7]. 10 antiparallel $\beta$ sheets form a barrel, which is covered by a lid-like loop of two $\alpha$-helixes [8]. Inside the barrel, there is a cocrystallized lipid from E.coli.

The role of P2 in myelin is unclear, although as a FABP, it might be involved in lipid transfer between membrane layers. In myelin, P2 is located between two membrane bilayers binding to the polar heads of lipids by the positively charged loops at the top and bottom of the

${ }^{a}$ Corresponding author: saara.laulumaa@oulu.fi protein [7-9]. The lid is likely to penetrate the membrane; binding to lipids unfolds the $\alpha$-helices of the lid $[7,10]$.

P2 is known to stack lipid bilayers together $[7,11]$ and stabilize the membrane structure [12]. Previous neutron scattering studies have shown that $\mathrm{P} 2$ bound to myelinmimicking vesicles made either of 1,2-dimyristoyl-snglycero-3-phosphatidic acid (DMPA) or of a binary mixture of 1,2-dioleoyl-sn-glycero-3-phospho-L-serine (DOPS) and 1,2-dioleoyl-sn-glycero-3-phosphocholine (DOPC), respectively, reduces the lipid dynamics [13].

Understanding protein dynamics is crucial for studying protein structure-function relationships [14]. The protein atoms face different motions in a wide time scale from fast local atom vibrations to subdomain transitions and slow protein folding processes. Neutron scattering is a powerful technique for the investigation of biological macromolecules, since they contain approximately $50 \%$ hydrogen atoms that have a very large incoherent crosssection compared to other elements. Neutron scattering allows the investigation of molecular motions occurring on a pico- to nanosecond timescale and on an atomic lengthscale.

Here, we describe the dynamics of the P2 protein, mainly associated to local protons sitting on side chains, at the 10 -ps time scale, measured at $70-\mu \mathrm{eV}$ resolution on the IN6 time-of-flight instrument at ILL.

We measured the elastic incoherent scattering (EINS) of wild-type $\mathrm{P} 2$ and its proline 38 to glycine (P38G) point mutated form in a hydrated state. This point mutation of an amino acid with the most rigid backbone to the most flexible one lies in a hinge region between the barrel and the lid of the protein (Fig. 1). It does not affect protein

This is an Open Access article distributed under the terms of the Creative Commons Attribution License 4.0, which permits unrestricted use, distribution, and reproduction in any medium, provided the original work is properly cited. 


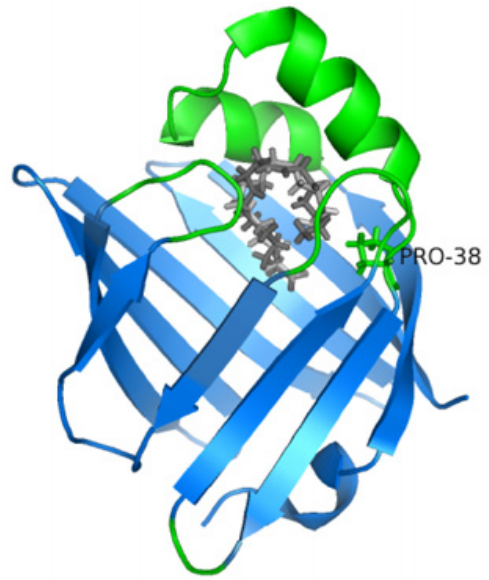

Figure 1. Crystal structure of human myelin protein P2 [8]. The point-mutated proline 38 lies at the hinge between the $\beta$-sheet barrel and $\alpha$-helical lid. Regions proposed to have increased flexibility due to the P38G mutation are shown in green color, and the bound fatty acid in gray.

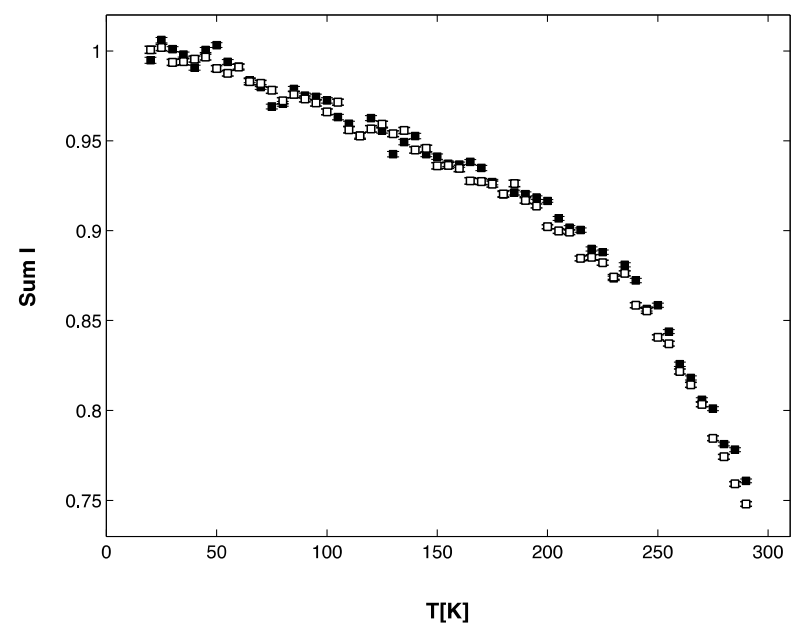

Figure 2. The sum of elastic intensities of wild-type (filled symbols) and P38G (empty symbols) P2 as a function of temperature.

folding or structure but increases the lipid-binding activity of the protein (unpublished data). Thus, we are observing the differences in total protein dynamics caused by the increased flexibility of the loops and lid that are crucial to protein-lipid interactions and protein activity.

\section{Materials and methods}

\subsection{Protein sample preparation}

Human myelin protein P2 was expressed recombinantly in E.coli Rosetta (DE3) cells using the pTH27 expression vector. In addition to the wild-type protein, the P38G mutant was prepared [15]. The proteins were expressed at $310 \mathrm{~K}$ and purified using Ni-ion affinity and size-exclusion chromatography, as described $[8,15]$.

The purified protein was dialyzed three times against $\mathrm{H}_{2} \mathrm{O}$ to carefully remove buffer traces from the sample and lyophilized. The achieved dry powder was then hydrated to $0.3 \mathrm{gD}_{2} \mathrm{O} / 1.0 \mathrm{~g}$ protein in a desiccator in a $\mathrm{N}_{2} / D_{2} \mathrm{O}$

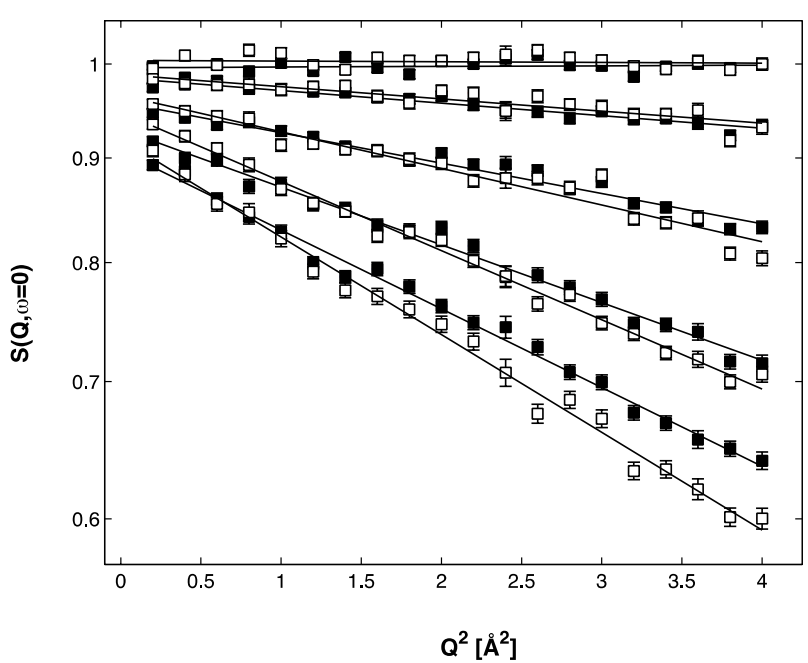

Figure 3. Gaussian fit of EINS data from wild-type P2 (filled symbols) and the P38G mutant (open symbols).

atmosphere. To achieve comparable wild type and mutant protein samples, they were prepared in parallel.

\subsection{Elastic incoherent neutron scattering}

Elastic neutron scattering experiments were performed at the time-focusing time-of-flight spectrometer IN6 (ILL, Grenoble, France), with an incident wavelength $\lambda=5.1 \AA$ and an energy resolution of $70 \mu \mathrm{eV}$ FWHM. The setting allows to access the momentum transfer range $0.3<\mathrm{Q}<$ $2 \AA^{-1}$.

Spectra were collected over a wide temperature range $(20-300 \mathrm{~K})$. The elastic scattered intensity was corrected for the empty cell contribution, and it was normalized with respect to the lowest-temperature scan $(\mathrm{T}=20 \mathrm{~K})$, to compensate for spurious background contributions and detector efficiency.

In order to avoid corrections from multiple scattering contributions, the cell thickness and geometry were properly chosen to minimize neutron absorption by the sample. A typical transmission percentage of $90 \%$ was guaranteed using standard $0.4 \mathrm{~mm}$ thick aluminium flat sample holders.

\section{Results and discussion}

A first visual inspection of the raw data employs the integration of the elastic intensities over the whole Q-range exploitable on IN6 as a function of temperature (Fig. 2). The EINS intensity is slightly lower for the mutated protein compared to the wild-type protein at temperatures above $\sim 190 \mathrm{~K}$. This gives a preliminary indication of enhanced proton mobility.

The elastic intensity as a function of $Q^{2}$ as fitted using eq. 1 (see theoretical section) within the Q-range obeying the limit of validity of the Gaussian approximation (Fig. 3) $[16,17]$. The resulting mean square displacements (MSD) are reported in Fig. 4 as a function of temperature.

For a deeper insight into the neutron scattering data, the MSDs were then analyzed in terms of a model presented by Zaccai $[14,18]$, which assumes the atomic 
Table 1. The parameters obtained using the force constant model.

\begin{tabular}{|c|c|c|}
\hline & Wild type & P38G mutant \\
\hline$\langle k\rangle(\mathrm{N} / \mathrm{m})$ & $2.6 \pm 0.1$ & $2.7 \pm 0.3$ \\
\hline$\left\langle k^{\prime}\right\rangle(\mathrm{N} / \mathrm{m})$ & $0.46 \pm 0.04$ & $0.32 \pm 0.02$ \\
\hline$\theta(\mathrm{K})$ & $110 \pm 15$ & $100 \pm 20$ \\
\hline$T_{0}(\mathrm{~K})$ & 234 & 245 \\
\hline$\Delta G_{0}(\mathrm{kcal} / \mathrm{mol})$ & 1.03 & 1.06 \\
\hline
\end{tabular}

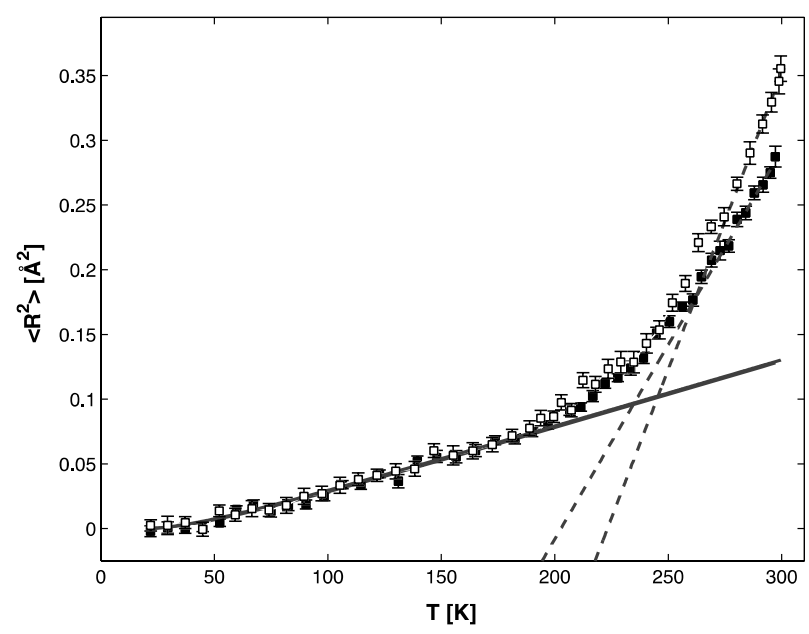

Figure 4. Mean square displacements of wild-type (filled symbols) and P38G P2 (open symbols) protein using Gaussian approximation. The continuous and dotted lines represent the fits using Eq. (2) and Eq. (3), respectively.

motion in macromolecules to be fluctuating within the energy conformational landscape, mainly between two classes of cages, in which the atoms accomplish small and large amplitude motions with respect to their equilibrium positions (see Theory). Thus, the MSDs were fitted using Eq. (2) at $\mathrm{T} \leq 150 \mathrm{~K}$, where dynamics is purely harmonic on IN6, and (3) at T $=280-300 \mathrm{~K}$, close to human body temperature $(310 \mathrm{~K})$. The resulting fitting parameters are listed in Table 1.

The fast local harmonic vibrational dynamics (small cage) are the same for both the wild type and P38G mutant samples within the error, with force constants $\langle k\rangle$ and Debye temperature $\theta \approx 105 \mathrm{~K}$. $\theta$ corresponds to a vibration frequency $\omega_{0} \approx 40 \mathrm{~cm}^{-1} \cdot \theta / 2=55 \mathrm{~K}$ is a temperature, above which the harmonic contribution changes linearly.

The transition temperatures $\left(T_{0}\right)$ are defined from Fig. 4. from the intersection of harmonic $\langle k\rangle$ and anharmonic $\left\langle k^{\prime}\right\rangle$ MSD curves. The P38G mutation of P2 increases $T_{0}$ by $\approx 10 \mathrm{~K}$. This stands for the activation energy $\Delta G_{0}$ which describes the free energy difference between the two cages of dynamics, being the same for wild-type and mutant P2 within the error. Simultaneously, the MSD of the mutant at $T_{0}$ is higher, indicating enhanced flexibility of the mutated protein. According to definition, at $T_{0}, 10 \%$ of the atoms are fluctuating in the large cage. This means that in the mutated protein, the amplitude of large cage dynamics is higher.

The same phenomenon can be seen from the pseudo force constant $\left\langle k^{\prime}\right\rangle$ at high temperatures: $\left\langle k^{\prime}\right\rangle$ for the P38G mutant is reduced by roughly $30 \%$ compared to wild-type P2. $\left\langle k^{\prime}\right\rangle$ describes the softness of a protein at physiological temperatures, corresponding to the force needed for an atom to fluctuate from its equilibrium position. Thus, a reduced $\left\langle k^{\prime}\right\rangle$ indicates an increasing average displacement of atoms in P38G, when the same energy is applied to both proteins.

\section{Conclusions}

The knowledge of protein dynamics is important to fully understand the functioning of macromolecules in biological systems [14]. The effect of point mutations on the protein dynamics has been studied before to a lower extend using EINS [19,20]; the difference in dynamics of wild type and mutated bacteriorhodopsin in purple membrane was not observable [19], whereas in solution, the mutated nonfunctional photosynthetic reaction center was shown to be more dynamic compared to wild type protein complex [20]. Here, we have studied the dynamics of hydrated protein powders using very pure myelin protein $\mathrm{P} 2$ samples.

P2 has an ability to bind phospholipids and stack membranes together. In our studies on a line of point mutant variants of human P2 [15], P38G has been identified as a "hyperactive" variant, being even more efficient than the wild-type protein in lipid membrane binding (manuscript in preparation). This simple point mutation does not affect the folding, surface structure, or charge of a protein; it does not bring any new binding sites to interact with phospholipids. Proline is an amino acid with the most rigid backbone, and it is often found in places, where the protein main chain makes sharp turns. In P2, Pro38 is between the stable barrel and the lid. In the P38G mutant protein, this proline is replaced by glycine, with the most flexible backbone. This is likely to increase the dynamics of the $\alpha$-helical lid and the nearby loops that interact with phospholipid membranes and the fatty acid inside the barrel (Fig. 1) [7].

We have shown that the local harmonic vibrations are similar for wild-type and mutated P2. The anharmonicity of the protein starts at $180 \mathrm{~K}$, where MSD differs from the small cage dynamics contribution. At physiological temperatures, the $\mathrm{P} 38 \mathrm{G}$ mutation of $\mathrm{P} 2$ softens the protein structure and, thus, increases the average mean square displacement of atoms at the 10-ps timescale.

\section{Theory}

We used a model presented by Zaccai [14,18], where the dynamical transition from harmonic to anharmonic motions, force constants and parameters of the energy landscape can be defined.

The elastic intensity as a function of $\mathrm{Q}^{2}$ can be fitted using a Gaussian model

$$
I(Q, \omega=0)=I_{0} e^{-Q^{2}\left(\Delta x^{2}\right)},
$$

where $\left\langle\Delta x^{2}\right\rangle=\frac{1}{3}\left\langle R^{2}\right\rangle$ and $\left\langle R^{2}\right\rangle$ is the three-dimensional vector of MSD, containing all kinds of local dynamic fluctuations at the 10-ps time scale. 
$\langle R\rangle^{2}$ can be determined as dynamics in two different conformational cages, where atoms are fluctuating with small or large amplitudes Assuming the small cage dynamics corresponds to vibrational harmonic oscillation, at low temperatures

$$
\left\langle R^{2}\right\rangle=\frac{k_{B} \theta}{2\langle k\rangle} \operatorname{cotanh}\left[\frac{\theta}{2 T}\right],
$$

where $\theta=\hbar \omega_{0} / k_{B}$ is the Debye temperature with an oscillation frequency $\omega_{0}$ and $\langle k\rangle$ is a force constant of oscillation.

Increasing the temperature, atoms will get freedom to fluctuate anharmonically with a larger amplitude. From the slope of the increasing MSD, one can determine the effective pseudo force constant $\left\langle k^{\prime \prime}\right\rangle$, describing the force of molecule dynamics at biologically relevant temperatures $\mathrm{T} \approx 300 \mathrm{~K}$, including atoms moving in both the small and large cages. Thus, the molecule resilience $\left\langle k^{\prime}\right\rangle$ is calculated as

$$
\left\langle k^{\prime}\right\rangle=\frac{k_{B} T}{d\left\langle R^{2}\right\rangle / d T}
$$

where $d T$ is a temperature region close to physiological temperatures. From the intersection of harmonic and anharmonic motion contributions, $T_{0}$, a transition temperature, where $10 \%$ of atoms $(\phi(T)=0.1)$ are fluctuating with a large amplitude [18], can be determined. $T_{0}$ is strongly dependent on the energy difference between the conformational cages. It is an optimal parameter for comparing molecular dynamics indifferent systems or/and in different environmental conditions. Because $\phi(T)=1 /\left(1+e^{\beta \Delta G}\right)$ with $\beta=(R T)^{-1}$, the free energy difference for the system at $T_{0}$ is

$$
\Delta G_{0}=\ln (9) R T_{0} .
$$

We acknowledge the IN6 instrument scientist Michael Koza for his support with the experiment.

\section{References}

[1] P. Kursula, Amino Acids 34, 175-185 (2008)

[2] H. Han, M. Myllykoski, S. Ruskamo, C. Wang, P. Kursula, Biofactors 39, 233-241 (2013)

[3] S. König, T. M. Bayerl, G. Coddens, D. Richter, E. Sackmann, Biophys. J. 68, 1871-1880 (1995)
[4] R. H. Quarles, W. B. Macklin, P. Morell, Basic Neurochemistry: Molecular, Cellular and Medical Aspects, 51-71 (Academic Press Elsevier, New York, 2006)

[5] J. Patzig, O. Jahn, S. Tenzer, S. P. Wichert, P. de Monasterio-Schrader, S. Rosfa, J. Kuharev, K. Yan, I. Bormuth, J. Bremer, A. Aguzzi, F. Orfaniotou, D. Hesse, M. H. Schwab, W.Möbius, K.-A. Nave, H. B. Werner, J. Neurosci. 31(45), 16369-16386 (2011)

[6] P. Morell, R. H. Quarles,W. T. Norton, Basic neurochemistry: molecular, cellular, and medical aspects, 117-143 (Raven, New York, 1994)

[7] S. Ruskamo, R.P. Yadav, S. Sharma, M. Lehtimäki, S. Laulumaa, S. Aggarwal, M. Simons, J. Bürck, A.S.Ulrich, A.H. Juffer, I. Kursula, P. Kursula, Acta Cryst. D 70, 165-176 (2014)

[8] V. Majava, E. Polverini, A. Mazzini, R. Nanekar, W. Knoll, J. Peters, F. Natali, P. Baumgärtel, I. Kursula, P. Kursula, PLoS One 5, e10300 (2010)

[9] B. D. Trapp, M. Dubois-Dalcq and R. H. Quarles, J. Neurochem. 43(4), 944-948 (1984)

[10] P. Riccio, F. Zito, A. Fasano, G. M. Liuzzi, F. Lolli, E. Polverini and P. Cavatorta, NeuroReport 9, 27692773 (1998)

[11] S. Suresh, C. Wang, R. Nanekar, P. Kursula \& J.M. Edwardson, Biochemistry 49, 3456-3463 (2010)

[12] W. Knoll, J. Peters, P. Kursula, Y. Gerelli, J. Ollivier, B. Deme, M. Telling, E. Kemner, F. Natali, Soft Matter 10, 519-529 (2014)

[13] W. Knoll, F. Natali, J. Peters, R. Nanekar, C. Wang, P. Kursula, Spectroscopy 24(6), 585-592 (2010)

[14] G. Zaccai, Science 288, 1604-1607 (2000)

[15] M. Lehtimäki, S. Laulumaa, S. Ruskamo, P. Kursula, Acta Cryst. F 68, 1359-1362 (2012)

[16] W. Doster, S. Cusack, W. Petry, Nature 337, 754-756 (1989).

[17] V. Reat, G. Zaccai, M. Ferrand, C. Pfister, Biological Macromolecular Dynamics 117-121 (Adenine Press, Schenectady, NY, 1997)

[18] D. J. Bicout, and G. Zaccai, Biophys. J. 80, 11151123 (2001)

[19] U. Lehnert, V. Réat, G. Zaccai, D. Oesterhelt, Eur Biophys J. 34, 344-352 (2005)

[20] S. Sacquin-Mora, P. Sebban, V. Derrien, B. Frick, R. Lavery, C. Alba-Simionesco, Biochemistry 46, 14960-14968 (2007) 